\title{
Use of a Metric in Supervisory Control of Probabilistic Discrete Event Systems
}

\author{
Vera Pantelic and Mark Lawford \\ SQRL, Department of Computing and Sofware, Faculty of \\ Engineering, McMaster University, Hamilton, ON, Canada L8S 4 K1 \\ (e-mail: pantelv at mcmaster dot ca, lawford at mcmaster dot ca)
}

\begin{abstract}
This work represents a natural extension of our work on optimal probabilistic supervisory control of probabilistic discrete event systems (PDESs). In that work, a pseudometric on the initial states of two probabilistic automata that represent probabilistic systems is used to measure the distance between two systems. The pseudometric is given a fixed point characterization. This paper gives a logical characterization of the same pseudometric that justifies the intuition that two systems are close if they satisfy similar properties. A trace characterization of the pseudometric is then derived from the logical characterization. Further, the solution of the problem of approximation of a given probabilistic automaton with another automaton is suggested such that the new model is as close as possible to the original one in the pseudometric. The significance of the approximation is then discussed.
\end{abstract}

Keywords: Supervisory control, stochastic systems, discrete event systems, metrics.

\section{INTRODUCTION}

A supervisory control framework of PDESs was proposed in Lawford and Wonham (1993). PDESs are modeled as probabilistic generators from Garg (1992a,b). Further, deterministic supervisors for DES are generalized to probabilistic supervisors: after observing a string $s$, the probabilistic supervisor enables an event $\sigma$ with a certain probability. The supervisory control problem considered in Lawford and Wonham (1993) is to find, if possible, a supervisor under whose control the behaviour of a plant is identical to a given probabilistic specification. Further, Lawford and Wonham (1993) show that a plant under probabilistic control can generate a much larger class of probabilistic languages than deterministic control, and give the necessary and sufficient conditions for the existence of a supervisor for a class of PDESs. A formal proof of the necessity and sufficiency of the conditions and an algorithm for the calculation of the supervisor, if it exists, are presented in Postma and Lawford (2004), Pantelic et al. (2009). Analogous to a problem in classical supervisory control theory, it can happen that, given a plant to be controlled and a probabilistic specification language, no probabilistic supervisor exists such that the plant under control generates the prespecified probabilistic language. In this case when the exact solution is not achievable, a designer tries to find a supervisor such that the plant generates the behavior closest to the desired behaviour Pantelic and Lawford (2009). Therefore, the supervisor is synthesized by minimizing the distance between the achievable probabilistic behavior of the plant under control and the probabilistic behaviour of the requirement. The distance is measured by a pseudometric on the states of probabilistic transition systems which was introduced in Deng et al. (2006). The pseudometric is characterized as the greatest fixed point of a function. For reactive systems, the work of Deng et al. (2006) is closely related to Desharnais et al. (2002), Desharnais et al. (2004), van Breugel and Worrell (2001a). The pseudometric of Desharnais et al. (2004) is given a fixed point characterization, while the pseudometric of Desharnais et al. (2002) is given both logical and fixed point characterization. Further, van Breugel and Worrell $(2001 \mathrm{~b}, 2005)$ give a coalgebraic characterization of a pseudometric and show that it coincides with Desharnais et al. (2004).

The pseudometric of Deng et al. (2006) is slightly modified in Pantelic and Lawford (2009) and intuitively matches a notion of the distance between PDESs, accounting for all differences between corresponding transition probabilities. Furthermore, as the pseudometric is suggested for a large class of systems, it allows for an extension of the work to e.g., nondeterministic systems. Also, there is a simple algorithm to compute distances in this pseudometric for our generative, deterministic model (Pantelic and Lawford (2009)).

This paper further motivates the choice of the pseudometric in the solution of control problem of Pantelic and Lawford (2009) by characterizing the pseudometric using a logic along the lines of Desharnais et al. (2002). However, the logic itself is different than that of Desharnais et al. (2002) as our models are generative. Also, the main part of the characterization proof is, to the best of our knowledge, novel. The idea of logical characterization is that the distance between two systems is measured by a logical formula that distinguishes between the systems the most. Further, in this paper, this logical characterization is used as useful information on how the probabilistic traces of the systems (whose distance is measured by the pseudometric) are related. 
In control theory of PDESs, Chattopadhyay and Ray (2008) introduce a metric (in a symbolic pattern recognition application) to measure the distance between the original model and the one with prespecified structure, where the latter has the same long term distribution over the states as the original one. In this paper, the problem of a similar probabilistic model transformation (probabilistic model fitting) is discussed in our setting. A probabilistic generator is approximated with another one with a prespecified structure such that the distance between the two is as small as possible in the metric of Pantelic and Lawford (2009). The significance of the fitting, especially with respect to the control algorithm of Pantelic and Lawford (2009) is then discussed.

In Section 2, PDESs as generators of probabilistic languages are presented, and the problem of optimal probabilistic control of PDESs and its solution are introduced. Section 3 presents the logical characterization of the pseudometric. The trace characterization that stems from the logical one is presented in Section 4. The probabilistic model fitting problem, its solution, and its applications in control theory are introduced in Section 5. Section 6 concludes with avenues for future work.

All the proofs can be found in Appendix A.

\section{PRELIMINARIES}

In this section, PDESs modeled as generators of probabilistic languages are presented. Then, the problem of optimal supervisory control of PDESs, and the pseudometric used in the solution of the problem are introduced (for more details, see Pantelic and Lawford (2009)).

\subsection{Modeling PDES}

The probabilistic DES (PDES) can be modeled as a probabilistic generator $G=\left(Q, \Sigma, \delta, q_{0}, p\right)$ (Lawford and Wonham (1993)), where $Q$ is the nonempty finite set of states, $\Sigma$ is a finite alphabet whose elements we will refer to as event labels, $\delta: Q \times \Sigma \rightarrow Q$ is the (partial) transition function, $q_{0} \in Q$ is the initial state, and $p: Q \times \Sigma \rightarrow$ $[0,1]$ is the statewise event probability distribution. The results to be presented are for prefix closed probabilistic specification languages; hence the lack of marking states in the definition of a probabilistic generator. The transition function is traditionally extended by induction on the length of strings to $\delta: Q \times \Sigma^{*} \rightarrow Q$ in a natural way. For a state $q$, and a string $s$, the expression $\delta(q, s)$ ! will denote that $\delta$ is defined for the string $s$ in the state $q$.

The probability that the event $\sigma \in \Sigma$ is going to occur at the state $q \in Q$ is $p(q, \sigma)$. For the generator $G$ to be welldefined, $(i) p(q, \sigma)=0$ should hold if and only if $\delta(q, \sigma)$ is undefined, and $(i i) \forall q \sum_{\sigma \in \Sigma} p(q, \sigma) \leq 1$. The probabilistic generator $G$ is nonterminating if, for every reachable state $q \in Q, \sum_{\sigma \in \Sigma} p(q, \sigma)=1$. Conversely, $G$ is terminating if there is at least one reachable state $q \in Q$ such that $\sum_{\sigma \in \Sigma} p(q, \sigma)<1$. The probability that the system terminates at state $q$ is $1-\sum_{\sigma \in \Sigma} p(q, \sigma)$. Throughout the sequel, we will mostly consider nonterminating generators (if a plant is terminating, it can easily be transformed into a nonterminating one using the technique described in Lawford and Wonham (1993)).
The language $L(G)$ generated by a probabilistic DES automaton $G=\left(Q, \Sigma, \delta, q_{0}, p\right)$ is $L(G)=\left\{s \in \Sigma^{*} \mid \delta\left(q_{0}, s\right) !\right\}$. The probabilistic language generated by $G$ is defined as:

$$
\begin{aligned}
& L_{p}(G)(\epsilon)=1 \\
& L_{p}(G)(s \sigma)=\left\{\begin{array}{cc}
L_{p}(G)(s) \cdot p\left(\delta\left(q_{0}, s\right), \sigma\right) & \text { if } \delta\left(q_{0}, s\right) ! \\
0 & \text { otherwise }
\end{array}\right.
\end{aligned}
$$

Informally, $L_{p}(G)(s)$ is the probability that the string $s$ is executed in $G$. Also, $L_{p}(G)(s)>0$ iff $s \in L(G)$.

For each state $q \in Q$, function $\rho_{q}: \Sigma \times Q \rightarrow[0,1]$ is defined such that for any $q^{\prime} \in Q, \sigma \in \Sigma$, we have $\rho_{q}\left(\sigma, q^{\prime}\right)=p(q, \sigma)$ if $q^{\prime}=\delta(q, \sigma)$, and 0 otherwise. The function $\rho_{q}$ is a probability distribution on the set $\Sigma \times Q$. Also, for a state $q$, the set of possible events is defined to be $\operatorname{Pos}(q):=\{\sigma \in \Sigma \mid \delta(q, \sigma) !\}$.

\subsection{Control Problem}

As usually, the set $\Sigma$ is partitioned into disjoint sets $\Sigma_{c}$ and $\Sigma_{u}$, the sets of controllable and uncontrollable events, respectively. Deterministic supervisors for DES are generalized to probabilistic supervisors. Instead of deterministically enabling or disabling controllable events, probabilistic supervisors enable them with certain probabilities.

Optimal Probabilistic Supervisory Control Problem (OP$S C P)$ : Let $G_{1}=\left(Q, \Sigma, \delta_{1}, q_{0}, p_{1}\right)$ be a plant PDES, and let $G_{2}=\left(R, \Sigma, \delta_{2}, r_{0}, p_{2}\right)$ be a requirements specification PDES. If there is no probabilistic supervisor $V_{p}$ such that $L_{p}\left(V_{p} / G_{1}\right)=L_{p}\left(G_{2}\right)$ (i.e., the conditions of Lawford and Wonham (1993) fail), find $V_{p}$ such that

(1) $L\left(V_{p} / G_{1}\right) \subseteq L\left(G_{2}\right)$ and supervisor $V_{p}$ is maximally permissive in the nonprobabilistic sense (i.e., $L\left(V_{p} / G_{1}\right)$ is the supremal controllable sublanguage of $L\left(G_{1}\right) \cap L\left(G_{2}\right)$ with the respect to $\left.G_{1}\right)$.

(2) The probabilistic behaviour of the controlled plant is "as close as possible" to the probabilistic behaviour of the requirements specification, now restricted to supremal controllable sublanguage of $L\left(G_{1}\right) \cap L\left(G_{2}\right)$ with the respect to $G_{1}$.

The solution to the problem is given in Pantelic and Lawford (2009). The pseudometric used as a measure of proximity of the controlled plant and probabilistic requirements specification will be described in Section 2.3. Also, in Pantelic and Lawford (2009), an iterative algorithm is suggested to approximate the probabilities of controlled plant. The algorithm minimizes the distance between the controlled plant and the modified requirements specification such that the probabilistic controllability conditions of Lawford and Wonham (1993) are satisfied.

\subsection{The pseudometric}

Probabilistic bisimulation as commonly used to define an equivalence relation between probabilistic systems is not a robust relation: two states of probabilistic systems are bisimilar if and only if they have the same transitions with exactly the same probabilities to states in the same equivalence classes. As a more flexible way to compare probabilistic systems, a notion of pseudometric is introduced. A pseudometric on a set of states $Q$ is a function $d: Q \times Q \rightarrow$ $\mathbb{R}$ that defines a distance between two elements of $Q$, and 
satisfies the following conditions: $d(x, y) \geq 0, d(x, x)=0$, $d(x, y)=d(y, x)$, and $d(x, z) \leq d(x, y)+d(y, z)$, for any $x, y, z \in Q$. If all distances are not greater than 1 , the pseudometric is 1-bounded. In the sequel, the terms metric and pseudometric will be used interchangeably.

The work of Deng et al. (2006) introduces a pseudometric on states for a large class of probabilistic automata, including reactive and generative probabilistic automata. The pseudometric is based on the Kantorovich metric on distributions. Two states are at distance 0 in this metric if and only if they are probabilistic bisimilar. Here, the metric is presented only for probabilistic generators.

Let $G=\left(Q, \Sigma, \delta, q_{0}, p\right)$ be a nonterminating PDES, where $Q=\left\{q_{0}, q_{1}, \ldots q_{N-1}\right\}$. First, in Desharnais et al. (2002) and Deng et al. (2006), the class $\mathcal{M}$ of 1-bounded pseudometrics on states is defined with the ordering

$$
d_{1} \preceq d_{2} \text { if } \forall s, t d_{1}(s, t) \geq d_{2}(s, t) .
$$

Further, it is proved that $(\mathcal{M}, \preceq)$ is a complete lattice.

Next, let $d \in \mathcal{M}$, and let the constant $e \in(0,1]$ be a discount factor that determines the degree to which the difference in the probabilities of farther transitions is discounted: the smaller the value of $e$, the greater the discount on future transitions. Let $q_{q}, q_{r} \in Q$, and let $\rho_{q_{q}}$ and $\rho_{q_{r}}$ be the distributions on $\Sigma \times Q$ induced by the states $q_{q}$ and $q_{r}$, respectively. Next, let $i\left(q_{q}, \sigma\right)=i$ such that $q_{i}=$ $\delta\left(q_{q}, \sigma\right)$ if $\delta\left(q_{q}, \sigma\right)$ !, and $i\left(q_{q}, \sigma\right)=0$, otherwise. Similarly, $j\left(q_{r}, \sigma\right)=j$ such that $q_{j}=\delta\left(q_{r}, \sigma\right)$ if $\delta\left(q_{r}, \sigma\right)$ !, and $j\left(q_{r}, \sigma\right)=0$, otherwise. For readability purposes, we will write $i$ instead of $i\left(q_{q}, \sigma\right)$, and $j$ instead of $j\left(q_{r}, \sigma\right)$. Further, we will write $\rho_{\sigma, i}$ instead of $\rho_{q_{q}}\left(\sigma, q_{i}\right)$, and, similarly, $\rho_{\sigma, j}^{\prime}$ instead of $\rho_{q_{r}}\left(\sigma, q_{j}\right)$. Then, the pseudometric on states $d_{f p}$ is given as the greatest fixed-point of the function $\mathcal{D}$ on $\mathcal{M}$, that, in the special case of probabilistic generators, can be shown to be (see Pantelic and Lawford (2009)):

$$
\begin{aligned}
& \mathcal{D}(d)\left(q_{q}, q_{r}\right) \\
& =\sum_{\sigma \in \Sigma} \max \left(\rho_{\sigma, i}-\rho_{\sigma, j}^{\prime}+e \rho_{\sigma, j}^{\prime} d\left(q_{i}, q_{j}\right), e \rho_{\sigma, i} d\left(q_{i}, q_{j}\right)\right) \\
& =\sum_{\sigma \in\left\{\sigma \in \Sigma \mid \rho_{\sigma, i} \geq \rho_{\sigma, j}^{\prime}\right\}}\left(\rho_{\sigma, i}-\rho_{\sigma, j}^{\prime}+e \rho_{\sigma, j}^{\prime} d\left(q_{i}, q_{j}\right)\right) \\
& +\sum_{\sigma \in\left\{\sigma \in \Sigma \mid \rho_{\sigma, i}<\rho_{\sigma, j}^{\prime}\right\}} e \rho_{\sigma, i} d\left(q_{i}, q_{j}\right)
\end{aligned}
$$

We arbitrarily choose $i\left(q_{q}, \sigma\right)$ to be 0 (similarly for $j\left(q_{r}, \sigma\right)$ ) when $\delta\left(q_{q}, \sigma\right)$ is not defined although we could have chosen any other $i \in\{1, \ldots, N-1\}$. This is because when $\delta\left(q_{q}, \sigma\right)$ ! does not hold, then $\rho_{\sigma, i\left(q_{q}, \sigma\right)}=0$ for any $i\left(q_{q}, \sigma\right) \in$ $\{0, \ldots, N-1\}$.

The proofs that the function defined by (2) is monotone on $\mathcal{M}$, and that it has the greatest fixed point follow straightforwardly from Desharnais et al. (2002). Also, the metric $d_{f p}$ is 1-bounded, and the distances between the states in $d_{f p}$ are larger by the factor $1 / e$ than the distances in metric defined in Deng et al. (2006).

The pseudometric $d_{f p}$ is defined on the states of a single PDES. The distance between two PDESs (with disjoint sets of states) is the distance between their initial states in a new PDES that represents the union of the PDESs defined in a natural way (Pantelic and Lawford (2009)).
In the sequel, the union will not be formalized as it does not change the distance between the states.

\section{LOGICAL CHARACTERIZATION}

The aforementioned metric has been given a fixed point characterization. This section presents a logical characterization of the same metric. The idea is as follows. Intuitively, when two systems are metrically similar, they should satisfy similar properties. If the systems are probabilistic bisimilar, they should satisfy the same properties. Further, the distance between the states in the metric is achieved through a formula that distinguishes between the systems the most.

As before, let $G=\left(Q, \Sigma, \delta, q_{0}, p\right)$ be a nonterminating generator, where $Q=\left\{q_{0}, q_{1}, \ldots q_{N-1}\right\}$.

Definition 1. Given an alphabet $\Sigma$, the logic $\mathcal{L}$ is defined as follows:

$$
\phi::=1|\langle\sigma\rangle \phi| \bigvee_{\sigma \in \Theta}\langle\sigma\rangle \phi|1-\phi| \phi \ominus p
$$

where $p$ is a rational number in $[0,1], \sigma \in \Sigma$, and $\Theta \subseteq \Sigma$.

The formula $\phi$ evaluated at a state $q \in Q, \phi(q)$, is a measure of how much $\phi$ is satisfied at the state. The semantics of the logic $\mathcal{L}$ is given next.

Definition 2. Let $q \in Q$, and $\rho_{q}$ be the probability distribution on $\Sigma \times Q$ induced by state $q$. Let $\phi \in \mathcal{L}$, and $\psi: \Sigma \rightarrow \mathcal{L}$. The notation $\psi_{\sigma}$ will be used for $\psi(\sigma), \sigma \in \Sigma$. Then:

$$
\begin{aligned}
\mathbf{1}(q) & =1 \\
\langle\sigma\rangle \phi(q) & =e \rho_{q}\left(\sigma, q_{i(q, \sigma)}\right) \phi\left(q_{i(q, \sigma)}\right) \\
\bigvee_{\sigma \in \Theta}\langle\sigma\rangle \psi_{\sigma}(q) & =\sum_{\sigma \in \Theta} e \rho_{q}\left(\sigma, q_{i(q, \sigma)}\right) \psi_{\sigma}\left(q_{i(q, \sigma)}\right) \\
(1-\phi)(q) & =1-\phi(q) \\
(\phi \ominus p)(q) & =\max (\phi(q)-p, 0)
\end{aligned}
$$

where $\sigma \in \Sigma$, and, as before, $i(q, \sigma)=i$ such that $q_{i}=\delta(q, \sigma)$ if $\delta(q, \sigma)$ !, and $i(q, \sigma)=0$, otherwise.

The presented logic represents a probabilistic modification of Hennessy-Milner logic (Hennessy and Milner (1985)). The formula 1 corresponds to the constant true, $\langle\sigma\rangle \phi$ is the next operator, $1-\phi$ corresponds to negation, and $\phi \ominus p$ provides for the testing of the value of $\phi$ (Desharnais et al. (2002)). The logic supports only a specific disjunction of form $\bigvee\langle\sigma\rangle \phi$; extending it to $\bigvee \phi$ would require a more complicated formalization not necessary for the main result to be presented.

The metric $d_{L}$ is next defined. Two states are measured by a formula that differentiates them the most in the following manner.

Definition 3. For every $q_{q}, q_{r} \in Q$, the metric $d_{L}$ is defined as:

$$
d_{L}\left(q_{q}, q_{r}\right)=\sup _{\phi \in \mathcal{L}}\left\{\left|\phi\left(q_{q}\right)-\phi\left(q_{r}\right)\right|\right\}
$$

In this logical setting, the smaller the factor $e$ is, the more discounted the difference is for complex formulae.

An example is given in Figure 1 . The states $q_{0}$ and $r_{0}$ are at the distance $0.25 e+0.75 e^{2}$ in the metric $d_{L}$, witnessed by the formula $\phi=\bigvee_{\sigma \in\{\alpha, \beta\}}\langle\sigma\rangle \phi_{\sigma}$, where $\phi_{\alpha}=1-\langle\gamma\rangle \mathbf{1}$, 

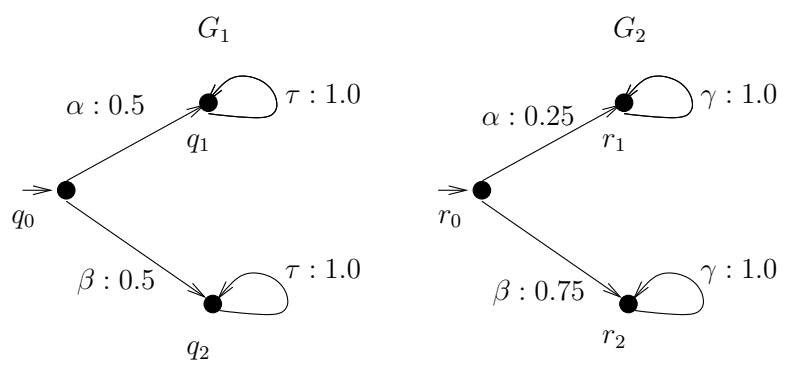

Fig. 1. Example

and $\phi_{\beta}=\langle\tau\rangle \mathbf{1}$. Further, states $q_{1}$ and $r_{1}$ (also, $q_{1}$ and $r_{2}$ ) are at the distance $e$ as witnessed by the formula $\phi=\langle\tau\rangle \mathbf{1}$.

The goal is to show that the metric $d_{f p}$ is equal to the metric $d_{L}$ up to constant $e$.

Lemma 1. Let $q_{q}, q_{r} \in Q$. For a function $\phi: \Sigma \rightarrow \mathcal{L}$, the shorthand notation $\phi_{\sigma}$ will be used for $\phi(\sigma)$. Then:

$$
d_{L}\left(q_{q}, q_{r}\right)=\sup _{\phi_{\sigma} \in \mathcal{L}}\left\{\left|\bigvee_{\sigma \in \Sigma}\langle\sigma\rangle \phi_{\sigma}\left(q_{q}\right)-\bigvee_{\sigma \in \Sigma}\langle\sigma\rangle \phi_{\sigma}\left(q_{r}\right)\right|\right\} .
$$

The following two definitions will be used for the proof of the main result. First, the depth of a formula $\phi \in \mathcal{L}$ is defined (in a manner similar to that of Desharnais et al. (2002)). Then, the formula $\phi_{q_{q}, q_{r}}^{n}$ is introduced.

Definition 4. The depth of a formula of logic $\mathcal{L}$ is defined as:

$$
\begin{aligned}
\operatorname{depth}(\mathbf{1}) & =0, \\
\operatorname{depth}(\langle\sigma\rangle \phi) & =\operatorname{depth}(\phi)+1, \\
\operatorname{depth}\left(\bigvee_{\sigma \in \Theta}\langle\sigma\rangle \psi_{\sigma}(q)\right) & =\max \left\{\operatorname{depth}\left(\psi_{\sigma}\right) \mid \sigma \in \Theta\right\}+1, \\
\operatorname{depth}(1-\phi) & =\operatorname{depth}(\phi), \\
\operatorname{depth}(\phi \ominus p) & =\operatorname{depth}(\phi) .
\end{aligned}
$$

Definition 5. Let $q_{q}, q_{r} \in Q$. The notation adopted for (2) is used here. Then, formula $\phi_{q_{q}, q_{r}}^{0}$ is defined as

$$
\phi_{q_{q}, q_{r}}^{0}=\mathbf{1}
$$

and, for $n \in \mathbb{N}$, formula $\phi_{q_{q}, q_{r}}^{n+1}$ is defined as

$$
\begin{gathered}
\phi_{q_{q}, q_{r}}^{n+1}=\bigvee_{\sigma \in \Sigma}\langle\sigma\rangle \psi_{\sigma, q_{q}, q_{r}}^{n}, \text { where } \\
\psi_{\sigma, q_{q}, q_{r}}^{n} \\
=\left\{\begin{array}{cc}
1-\left(\left(1-\phi_{q_{i}, q_{j}}^{n}\right) \ominus\left(1-\phi_{q_{i}, q_{j}}^{n}\left(q_{i}\right)\right)\right) & \text { if } \rho_{\sigma, i} \geq \rho_{\sigma, j}^{\prime} \\
\phi_{q_{i}, q_{j}}^{n} \ominus \phi_{q_{i}, q_{j}}^{n}\left(q_{j}\right) & \text { otherwise. }
\end{array}\right.
\end{gathered}
$$

Remark 1. Back to the metric $d_{f p}$, according to Tarski's fixed point theorem, the greatest fixed point of function $\mathcal{D}$ can be reached through an iterative process that starts from the greatest element. As the number of transitions from a state of a probabilistic generator is finite, the greatest fixed point of the function $\mathcal{D}$ is reached after at most $\omega$ iterations (Deng et al. (2006), Desharnais et al. (2002)) (equivalently, the closure ordinal of $\mathcal{D}$ is $\omega)$. Therefore, the metric $d_{f p}$ can be reached through the following iterative process.

Definition 6. The distance function $d_{f p}^{0}$ is defined as:

$$
d_{f p}^{0}=0,
$$

and the distance function $d_{f p}^{n+1}, n \in \mathbb{N}$, is given as:

$$
d_{f p}^{n+1}=\mathcal{D}\left(d_{f p}^{n}\right),
$$

where $\mathcal{D}$ is given in (2).

The main result relating the two metrics is next presented. It states that metrics $d_{L}$ and $d_{f p}$ are equal up to constant e.

Theorem 1. $d_{L}=e d_{f p}$

Remark 2. The logic $\mathcal{L}$ can be easily extended such that $d_{L}=e d_{f p}$ still holds. Therefore, it is easy to make the logic more expressive while preserving the same characterization of our logic. As logic $\mathcal{L}$ is sufficient for the characterization of the metric, and for the sake of simplicity of formalization, the logic was not extended.

\section{FROM LOGIC TO TRACES}

First, $L_{p}(G)(s)$ is modified to define discounted probability of a string $s$ in $G, P_{d}(G)(s)$.

Definition \%. Let $P_{d}(G): L(G) \rightarrow[0,1]$ be defined as:

$$
\begin{aligned}
& P_{d}(G)(\epsilon)=1 \\
& P_{d}(G)(s \sigma)=\left\{\begin{array}{cc}
e \cdot P_{d}(G)(s) \cdot p\left(\delta\left(q_{0}, s\right), \sigma\right) & \text { if } \delta\left(q_{0}, s\right) ! \\
0 & \text { otherwise }
\end{array}\right.
\end{aligned}
$$

where $s \in L(G), \sigma \in \Sigma$. Then, $P_{d}(G)(s)$ is the discounted probability of a string $s$ in $G$.

Informally, the discounted probability of a string is the probability of occurrence of a string discounted by factor $e$ for every event in the string.

Let $G_{1}$ and $G_{2}$ be two probabilistic generators. An important result states that there is not a string whose discounted probability differs between the two languages more than the distance $d_{L}$ between corresponding generators.

Theorem 2.

$$
d_{L}\left(G_{1}, G_{2}\right) \geq \sup _{s \in \Sigma^{*}}\left\{\left|P_{d}\left(G_{1}\right)(s)-P_{d}\left(G_{2}\right)(s)\right|\right\}
$$

Further, it can be shown that distance in the metric $d_{L}$ between the two systems is also greater than the difference in discounted probabilities of a set of strings such that none of strings is a substring of another. Let $\Gamma \subseteq \Sigma^{*}$, such that no string in $\Gamma$ is a prefix of another string in $\Gamma$. Then:

Theorem 3.

$$
d_{L}\left(G_{1}, G_{2}\right) \geq \sup _{\Gamma \subseteq \Sigma^{*}}\left\{\left|\sum_{s \in \Gamma} P_{d}\left(G_{1}\right)(s)-\sum_{s \in \Gamma} P_{d}\left(G_{2}\right)(s)\right|\right\}
$$

Similarly, the correspondence between the discounted probability of strings and formulae in $\mathcal{L}$ can be made for the remaining formulae of Definition 2. Therefore, the metric measures not only the difference in probabilities of strings in two languages (discounted for their length), but also the difference in discounted probabilities of a certain set of strings, or some more complicated properties of strings, e.g., whether the discounted probability of a string is greater than a prespecified value.

\section{PROBABILISTIC MODEL FITTING}

First, the problem of probabilistic model fitting is defined, and a solution for the fitting is suggested. Next, its significance in control theory, and especially, with regards to the algorithm of Pantelic and Lawford (2009), is discussed. 


\subsection{Probabilistic Model Fitting: Problem and Solution}

For a probabilistic generator $G=\left(Q, \Sigma, \delta, q_{0}, p\right)$, the (nonprobabilistic) discrete event system (DES) that underlies $G$ will be denoted $G^{\prime}=\left(Q, \Sigma, \delta, q_{0}\right)$. The synchronous product of two (nonprobabilistic) discrete event systems (DESs) that underlie two PDESs is defined as follows. Let $G_{1}=\left(Q_{1}, \Sigma, \delta_{1}, q_{01}, p_{1}\right)$ and $G_{2}=\left(Q_{2}, \Sigma, \delta_{2}, r_{0}, p_{2}\right)$ be probabilistic generators.

Definition 8. The synchronous product of $G_{1}^{\prime}=\left(Q_{1}, \Sigma\right.$, $\left.\delta_{1}, q_{01}\right)$ and $G_{2}^{\prime}=\left(Q_{2}, \Sigma, \delta_{2}, r_{0}\right)$, denoted $G_{1}^{\prime} \| G_{2}^{\prime}$, is the reachable sub-DES of DES $G_{a}=\left(Q_{a}, \Sigma, \delta, q_{0}\right)$, where $Q_{a}=Q_{1} \times Q_{2}, q_{0}=\left(q_{01}, r_{0}\right)$, and, for any $\sigma \in \Sigma, q_{i} \in Q_{i}$, $i=1,2$, it holds that $\delta\left(\left(q_{1}, q_{2}\right), \sigma\right)=\left(\delta_{1}\left(q_{1}, \sigma\right), \delta_{2}\left(q_{2}, \sigma\right)\right)$ whenever $\delta_{1}\left(q_{1}, \sigma\right)$ !, and $\delta_{2}\left(q_{2}, \sigma\right)$ !.

Note that no minimization is done in the construction of the synchronous product as defined above.

Probabilistic Model Fitting Problem: Let $G_{1}=\left(Q_{1}, \Sigma, \delta_{1}\right.$, $\left.q_{01}, p_{1}\right)$ be a probabilistic generator. Given a nonprobabilistic generator $G_{2}^{\prime}=\left(Q_{2}, \Sigma, \delta_{2}, r_{0}\right)$ such that $G_{1}^{\prime} \| G_{2}^{\prime}$ is isomorphic to $G_{2}^{\prime}$, find the statewise event probability distribution $p_{2}$ such that probabilistic generator $G_{2}=$ $\left(Q_{2}, \Sigma, \delta_{2}, r_{0}, p_{2}\right)$ is as close as possible to $G_{1}$ in the metric $d_{f p}$.

The idea of solving the problem is as follows. The generator $G_{1}$ is to be modified to make $G_{2}^{\prime}$ isomorphic (identical up to renaming of states) to a subautomaton of $G_{1}^{\prime}$, while the probabilistic language of $G_{1}$ is preserved. Then, the distance between $G_{1}$ and $G_{2}$ is minimized by minimizing the distance between the modified $G_{1}$, and $G_{2}$. This is allowed as the two distances are the same, since $G_{1}$ and its modified version are probabilistic bisimilar:

Theorem 4. Let $G_{1}$ and $G_{2}$ be two probabilistic generators. Then, if $L_{p}\left(G_{1}\right)=L_{p}\left(G_{2}\right)$, then $d_{f p}\left(G_{1}, G_{2}\right)=0$.

Next, as previously stated, we seek to represent $L_{p}\left(G_{1}\right)$ with an automaton $G_{1 a}$ such that $G_{2}^{\prime}$ is isomorphic to a subautomaton of $G_{1 a}^{\prime}$. Figure 2 illustrates an example. The part of $G_{1 a}$ drawn by a solid line corresponds to the subautomaton of $G_{1 a}^{\prime}$ isomorphic to $G_{2}^{\prime}$. In general, the automaton $G_{1 a}$ will represent a non-minimal realization of $L_{p}\left(G_{1}\right)$ (in the sense that it might have more states than $G_{1}$, but $\left.L_{p}\left(G_{1}\right)=L_{p}\left(G_{1 a}\right)\right)$. Generator $G_{1 a}$ can be constructed in the following manner.

(1) Self-loop each state of $G_{2}^{\prime}$ with events not possible from that state. Formally, $G_{2 a}^{\prime}=\left(Q_{2}, \Sigma, \delta_{2 a}, r_{0}\right)$, where, for $q \in Q_{2}, \sigma \in \Sigma$ :

$$
\delta_{2 a}(q, \sigma)=\left\{\begin{array}{cc}
\delta_{2}(q, \sigma) & \text { if } \delta_{2}(q, \sigma) ! \\
q & \text { otherwise }
\end{array}\right.
$$

(2) Next, let $G_{1 a}^{\prime}=\left(Q_{1 a}, \Sigma, \delta_{1 a}, q_{0}\right)=G_{1}^{\prime} \| G_{2 a}^{\prime}$.

(3) The probabilistic version of $G_{1 a}^{\prime}$ is $G_{1 a}=\left(Q_{1 a}, \Sigma, \delta_{1 a}\right.$, $\left.q_{0}, p_{1 a}\right)$, such that, for all $q \in Q_{1 a}, \sigma \in \Sigma$ :

$$
p_{1 a}(q, \sigma)=p_{1}(r, \sigma) \text {, }
$$

where $r=\delta_{1}\left(q_{01}, s\right)$ for any $s \in L\left(G_{1 a}\right)$ such that $q=\delta_{1 a}\left(q_{0}, s\right)$.

Theorem 5. $L_{p}\left(G_{1}\right)=L_{p}\left(G_{1 a}\right)$.

Now, let $f: Q_{2} \rightarrow Q_{1 a}$ be an embedding (a monomorphism) of $G_{2}^{\prime}$ into $G_{1 a}^{\prime}$, i.e.:
(1) $f\left(r_{0}\right)=q_{0}$,

(2) $\forall q \in Q_{2} \forall \sigma \in \operatorname{Pos}(q) f\left(\delta_{2}(q, \sigma)\right)=\delta_{1 a}(f(q), \sigma)$.

The function $f$ always exists and is unique. This fact follows from construction of $G_{1 a}$ and determinism of generators.

Without loss of generality, it is assumed that, $Q_{1 a}=$ $\left\{q_{0}, \ldots, q_{M-1}\right\}, Q_{2}=\left\{r_{0}, \ldots, r_{N-1}\right\}$, and $N, M>0$, $d \in \mathcal{M}, q \in Q_{2}$. Next, $i(f(q), \sigma)=i$ such that $q_{i}=$ $\delta_{1 a}(f(q), \sigma)$ if $\delta_{1 a}(f(q), \sigma)$ !, and $i(f(q), \sigma)=0$, otherwise. Let $j(q, \sigma)=j$ such that $r_{j}=\delta_{2}(q, \sigma)$ if $\delta_{2}(q, \sigma)$ !, and $j(q, \sigma)=0$, otherwise. For readability purposes, we will write $i$ instead of $i(f(q), \sigma)$, and $j$ instead of $j(q, \sigma)$. The distance between $G_{1 a}$ and $G_{2}$ is $d_{f p}\left(q_{0}, r_{0}\right)$. Also, $f\left(r_{0}\right)=q_{0}$, and

$$
\begin{aligned}
& \mathcal{D}(d)(f(q), q) \\
& =\sum_{\sigma \in \Sigma} \max \left(\rho_{\sigma, i}-\rho_{\sigma, j}^{\prime}+e \rho_{\sigma, j}^{\prime} d\left(q_{i}, r_{j}\right), e \rho_{\sigma, i} d\left(q_{i}, r_{j}\right)\right) \\
& =\sum_{\sigma \in \operatorname{Pos}(f(q)) \backslash \operatorname{Pos}(q)} \rho_{\sigma, i} \\
& +\sum_{\sigma \in \operatorname{Pos}(q)} \max \left(\rho_{\sigma, i}-\rho_{\sigma, j}^{\prime}+e \rho_{\sigma, j}^{\prime} d\left(f\left(r_{j}\right), r_{j}\right),\right. \\
& \left.e \rho_{\sigma, i} d\left(f\left(q_{j}\right), q_{j}\right)\right)
\end{aligned}
$$$$
\text { (since } f\left(r_{j}\right)=q_{i} \text {, by the definition of } f \text { ) }
$$

where $\rho_{f(q)}$ and $\rho_{q}$ are the distributions on $\Sigma \times Q$ induced by the states $f(q)$ and $q$, respectively, and $\rho_{\sigma, i}$ is written instead of $\rho_{f(q)}\left(\sigma, q_{i}\right)$, and, similarly, $\rho_{\sigma, j}^{\prime}$ instead of $\rho_{q}\left(\sigma, r_{j}\right)$.

Based on (5), it can be concluded that, for $q \in Q_{2}$, the distance between a state $f(q) \in Q_{1 a}$ and a state $q \in Q_{2}$, depends only on distances between $f(t)$ and $t, t \in Q_{2}$. In Figure 2, the distance between $G_{2}$ and $G_{1 a}$ depends only on distances between states of pairs $\left(q_{0}, r_{0}\right),\left(q_{1}, r_{1}\right)$, and $\left(q_{2}, r_{2}\right)$; states $q_{3}, q_{4}, q_{5}$ are irrelevant.

Therefore, in order to calculate the distance between $G_{1 a}$ and $G_{2}$, only the distances $d_{f p}(f(q), q), q \in Q_{2}$, are of interest. Therefore, the distance between $G_{1 a}$ and $G_{2}$, for a fixed $p_{2}$, can be found by at most $\omega$ iterations given in Definition 6, where the domain of $d_{f p}^{n}$ is restricted to $Q_{1 a} \times Q_{2}$ and only distances between $f(q) \in Q_{1 a}$ and $q \in Q_{2}$ are defined.

The solution of the probabilistic model fitting problem is presented next.

Theorem 6. Let $G_{1}=\left(Q_{1}, \Sigma, \delta_{1}, q_{01}, p_{1}\right)$ be a probabilistic generator. For given $G_{2}^{\prime}=\left(Q_{2}, \Sigma, \delta_{2}, r_{0}\right)$ (such that $G_{1}^{\prime} \|$ $G_{2}^{\prime}$ is isomorphic to $G_{2}^{\prime}$ ), the statewise event probability distribution $p_{2}$ such that $G_{2}=\left(Q_{2}, \Sigma, \delta_{2}, r_{0}, p_{2}\right)$ is as close as possible to $G_{1}$ in the metric $d_{f p}$ should satisfy, for all $r \in Q_{2}, \sigma \in \Sigma:$

$$
p_{2}(r, \sigma) \geq p_{1}(q, \sigma)
$$

where $q=\delta_{1}\left(q_{01}, s\right)$ for any $s \in L\left(G_{2}\right)$ such that $r=$ $\delta_{2}\left(r_{0}, s\right)$.

Therefore, the new model is not unique: as long as the probabilities of the events possible in the new model do not decrease, the new model is as close as possible to the original one. For the example from Figure 2, one of the possible solutions is represented by the rightmost generator of the figure. In another possible solution, the 

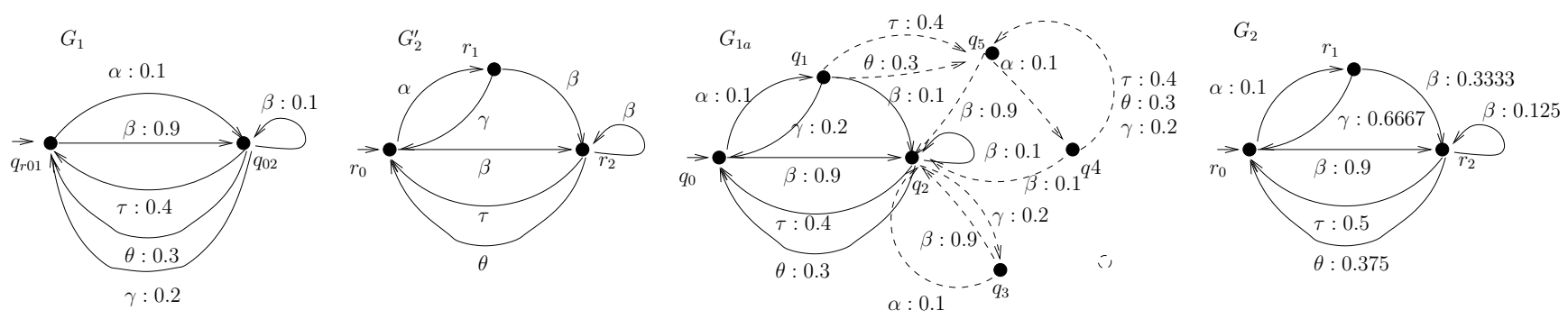

Fig. 2. Model fitting: an example

probabilities of occurrence of $\beta$ and $\gamma$ at the state $r_{1}$ would be 0.2 and 0.8 , respectively. Therefore, the fitting can be performed by any redistribution of the probabilities of events that are not possible anymore over the possible ones. Hence, model fitting can accommodate some further requirements on $p_{2}$.

\subsection{Applications of model fitting}

Other than the obvious use of the presented fitting to simplify and reduce the state space of probabilistic systems, the fitting has much more significant control implications.

As mentioned before, it is possible to choose probabilities of events in the new system to a certain extent: as long as they are greater or equal to the original ones. However, some of the further requirements on $p_{2}$ cannot be accommodated by Theorem 6 (e.g., an obvious one would be that the probability of an event still possible in the new system should be smaller than in the original system). If the restrictions are given on probabilities of events, statewise, a straightforward modification of the algorithm of Pantelic and Lawford (2009) for $e \in(0,1)$ would suffice. An example of such an additional requirement would be that the probability of a certain event from a state is less than a specified value, that is, in turn, smaller than the original one.

Further, in the solution of the optimal supervisory problem of Pantelic and Lawford (2009), in order for the first, maximal permissiveness requirement as presented in Section 2.2 to be satisfied, the supremal controllable sublanguage of $L\left(G_{1}\right) \cap L\left(G_{2}\right)$ with the respect to $G_{1}$ is generated. Then, the distance between the controlled plant, and the probabilistic requirement now restricted to the supremal controllable language, with normalized probabilities, is minimized. Intuitively, after satisfying the nonprobabilistic requirement, and before the probabilistic part is handled, it makes sense for a designer to modify the original requirement so that its nonprobabilistic behaviour matches the one achievable. Then, the probabilities are revised accordingly: probabilities of the events inadmissible for not satisfying the nonprobabilistic requirement are redistributed over the admissible ones. In Pantelic and Lawford (2009), the redistribution is such that the probability of an event in the new system is proportional to its original probability. Theorem 6 proves that this normalization is justified in a strict mathematical sense, as the new model that is normalized is as close as possible to the original one in the metric $d_{f p}$. However, a revised specification is going to be at a minimal distance from the original one as long as the probabilities of remaining events are greater or equal than the original ones: a designer has a freedom to choose how to redistribute the original probabilities of the events that are not possible in the new model over the ones that are still possible.

Further, the transformation of $G_{1}$ into $G_{1 a}$ presented here can be used in a modification of the algorithm of Pantelic and Lawford (2009) to handle the control problem of Pantelic and Lawford (2009) as presented in Section 2.2, with the requirement 2) changed so that the distance between the controlled plant and unmodified requirement is minimized. More precisely, the probabilistic language of requirements specification can be exactly represented using a probabilistic generator such that its underlying nonprobabilistic subautomaton is isomorphic to the generator representing supremal controllable language generated previously as explained in Section 2.2. Then, the algorithm of Pantelic and Lawford (2009) can be applied straightforwardly to minimize the distance between the two generators under probabilistic controllability conditions of Lawford and Wonham (1993).

\section{CONCLUSIONS}

A metric was used in a control algorithm to measure distance between two probabilistic generators in Pantelic and Lawford (2009). This paper gives a logical characterization of the metric that offers a better insight into the core of the metric from both logic and language standpoints. Further, the metric is used in the probabilistic model fitting problem: a probabilistic language is represented using a specified automaton structure such that that the new representation is as close as possible to the original one. Probabilistic model fitting has significant implications with respect to the work of Pantelic and Lawford (2009).

\section{REFERENCES}

Chattopadhyay, I. and Ray, A. (2008). Structural transformations of probabilistic finite state machines. International Journal of Control, 81(5), 820-835.

Deng, Y., Chothia, T., Palamidessi, C., and Pang, J. (2006). Metrics for action-labelled quantitative transition systems. Electronic Notes in Theoretical Computer Science, 153(2), 79-96. Also appeared in Proceedings of the 3rd Workshop on Quantitative Aspects of Programming Languages.

Desharnais, J., Gupta, V., Jagadeesan, R., and Panangaden, P. (2004). Metrics for labelled Markov processes. Theoretical Computer Science, 318(3), 323-354.

Desharnais, J., Jagadeesan, R., Gupta, V., and Panangaden, P. (2002). The metric analogue of weak bisimulation for probabilistic processes. In LICS '02: Proceedings of the 17th Annual IEEE Symposium on Logic 
in Computer Science, 413-422. IEEE Computer Society, Washington, DC, USA.

Garg, V. (1992a). An algebraic approach to modeling probabilistic discrete event systems. In Proceedings of 31st IEEE Conference on Decision and Control, 23482353. Tucson, AZ, USA.

Garg, V. (1992b). Probabilistic languages for modeling of DEDS. In Proceedings of 26th Conference on Information Sciences and Systems, volume 1, 198-203. Princeton, NJ.

Hennessy, M. and Milner, R. (1985). Algebraic laws for nondeterminism and concurrency. Journal of the ACM, $32(1), 137-161$.

Jou, C.C. and Smolka, S.A. (1990). Equivalences, congruences, and complete axiomatizations for probabilistic processes. In CONCUR, 367-383.

Lawford, M. and Wonham, W. (1993). Supervisory control of probabilistic discrete event systems. In Proceedings of the 36th IEEE Midwest Symposium on Circuits and Systems, volume 1, 327-331. IEEE.

Pantelic, V. and Lawford, M. (2009). Towards optimal supervisory control of probabilistic discrete event systems. In Proceedings of 2nd IFAC Workshop on Dependable Control of Discrete Systems. Bari, Italy.

Pantelic, V., Postma, S., and Lawford, M. (2009). Probabilistic supervisory control of probabilistic discrete event systems. Automatic Control, IEEE Transactions on, 54(8), 2013-2018.

Postma, S. and Lawford, M. (2004). Computation of probabilistic supervisory controllers for model matching. In Proceedings of Allerton Conference on Communications, Control, and Computing.

van Breugel, F. and Worrell, J. (2001a). An algorithm for quantitative verification of probabilistic transition systems. In K.G. Larsen and M. Nielsen (eds.), CONCUR, volume 2154 of Lecture Notes in Computer Science, 336350. Springer.

van Breugel, F. and Worrell, J. (2001b). Towards quantitative verification of probabilistic transition systems. In F. Orejas, P.G. Spirakis, and J. van Leeuwen (eds.), ICALP, volume 2076 of Lecture Notes in Computer Science, 421-432. Springer.

van Breugel, F. and Worrell, J. (2005). A behavioural pseudometric for probabilistic transition systems. Theoretical Computer Science, 331(1), 115-142.

\section{Appendix A. PROOFS}

\section{1) Proof of Lemma 1}

The idea of the proof is similar to that of Desharnais et al. (2002), Lemma 4.4. As before, for a function $\psi: \Sigma \rightarrow \mathcal{L}$, the shorthand notation $\psi_{\sigma}$ will be used for $\psi(\sigma)$. It should be proven that there exist $\psi_{\sigma} \in \mathcal{L}, \sigma \in \Sigma$, such that

$$
\left|\bigvee_{\sigma \in \Sigma}\langle\sigma\rangle \psi_{\sigma}\left(q_{q}\right)-\bigvee_{\sigma \in \Sigma}\langle\sigma\rangle \psi_{\sigma}\left(q_{r}\right)\right| \geq\left|\phi\left(q_{q}\right)-\phi\left(q_{r}\right)\right|
$$

for any $\phi \in \mathcal{L}$. Induction on the structure of $\phi$ is used. The base case $(\phi=1)$ is satisfied. Next, the case when $\phi=\langle\alpha\rangle \phi^{\prime}, \phi^{\prime} \in \mathcal{L}$, is investigated. It should be shown that

$$
\left|\bigvee_{\sigma \in \Sigma}\langle\sigma\rangle \psi_{\sigma}\left(q_{q}\right)-\bigvee_{\sigma \in \Sigma}\langle\sigma\rangle \psi_{\sigma}\left(q_{r}\right)\right| \geq\left|\langle\alpha\rangle \phi^{\prime}\left(q_{q}\right)-\langle\alpha\rangle \phi^{\prime}\left(q_{r}\right)\right| \text {. }
$$

If, for $\sigma \neq \alpha, \psi_{\sigma}=1-\mathbf{1}=0$, and $\psi_{\sigma}=\phi^{\prime}$ for $\sigma=\alpha$, the inequality is obviously satisfied. The case for $\phi=\bigvee_{\sigma \in \Theta}\langle\sigma\rangle \psi_{\sigma}$, for $\Theta \subseteq \Sigma$, is proven in the same manner.

The functions $\phi=1-\phi^{\prime}$ and $\phi=\phi^{\prime} \ominus p$ are non-expansive (easily shown), so

$$
\begin{aligned}
\left|\phi\left(q_{q}\right)-\phi\left(q_{r}\right)\right| & \leq\left|\phi^{\prime}\left(q_{q}\right)-\phi^{\prime}\left(q_{r}\right)\right| \\
& \leq\left|\bigvee_{\sigma \in \Sigma}\langle\sigma\rangle \psi_{\sigma}\left(q_{q}\right)-\bigvee_{\sigma \in \Sigma}\langle\sigma\rangle \psi_{\sigma}\left(q_{r}\right)\right|
\end{aligned}
$$

by induction on the structure of $\phi$.

2) Proof of Theorem 1

The proof consists of two parts. In the first part, it is proven that, for every $q_{q}, q_{r}$, there exists $\phi \in \mathcal{L}$ such that $\phi\left(q_{q}\right)-\phi\left(q_{r}\right)=e d_{f p}\left(q_{q}, q_{r}\right)$. Consequently, $d_{L}\left(q_{q}, q_{r}\right) \geq$ $e d_{f p}\left(q_{q}, q_{r}\right)$. In the second part, inequality $d_{L}\left(q_{q}, q_{r}\right) \leq$ $e d_{f p}\left(q_{q}, q_{r}\right)$ is proven.

First, let us prove that for every $q_{q}, q_{r}$, there exists $\phi \in \mathcal{L}$ such that $\phi\left(q_{q}\right)-\phi\left(q_{r}\right)=e d_{f p}\left(q_{q}, q_{r}\right)$. Given Remark 1 , it is sufficient to prove that $\phi_{q_{q}, q_{r}}^{n}\left(q_{q}\right)-\phi_{q_{q}, q_{r}}^{n}\left(q_{r}\right)=$ $e d_{f p}^{n}\left(q_{q}, q_{r}\right)$, for every $n \in \mathbb{N}$, where $\phi_{q_{q}, q_{r}}^{n}$ is given as in Definition 5 . The proof is by induction. The base case is trivial. Let assume that, for every $q_{q}, q_{r} \in Q, n \in \mathbb{N}$ :

$$
\phi_{q_{q}, q_{r}}^{n}\left(q_{q}\right)-\phi_{q_{q}, q_{r}}^{n}\left(q_{r}\right)=e d_{f p}^{n}\left(q_{q}, q_{r}\right) .
$$

Also, let $\rho_{q_{q}}$ and $\rho_{q_{r}}$ be the distributions on $\Sigma \times Q$ induced by the states $q_{q}$ and $q_{r}$, respectively. Also, for notational convenience, we will write $\rho_{\sigma, i}$ instead of $\rho_{q_{q}}\left(\sigma, q_{i}\right)$, and, similarly, $\rho_{\sigma, j}^{\prime}$ instead of $\rho_{q_{r}}\left(\sigma, q_{j}\right)$ for any $i, j$ such that $0 \leq i, j \leq N-1$. Then, for $\sigma \in \Sigma$, let $i\left(q_{q}, \sigma\right)=i$ such that $q_{i}=\delta\left(q_{q}, \sigma\right)$ if $\delta\left(q_{q}, \sigma\right)$ !, and $i\left(q_{q}, \sigma\right)=0$, otherwise. Similarly, let $j\left(q_{r}, \sigma\right)=j$ such that $q_{j}=\delta\left(q_{r}, \sigma\right)$ if $\delta\left(q_{r}, \sigma\right)$ !, and $j\left(q_{r}, \sigma\right)=0$, otherwise. For readability purposes, we will write $i$ instead of $i\left(q_{q}, \sigma\right)$, and $j$ instead of $j\left(q_{r}, \sigma\right)$. Then:

$$
\begin{aligned}
& \phi_{q_{q}, q_{r}}^{n+1}\left(q_{q}\right)-\phi_{q_{q}, q_{r}}^{n+1}\left(q_{r}\right) \\
& =\left(\sum_{\sigma \in\left\{\sigma \in \Sigma \mid \rho_{\sigma, i} \geq \rho_{\sigma, j}^{\prime}\right\}} e \rho_{\sigma, i}+\sum_{\sigma \in\left\{\sigma \in \Sigma \mid \rho_{\sigma, i}<\rho_{\sigma, j}^{\prime}\right\}} e \rho_{\sigma, i} e d_{f p}^{n}\left(q_{i}, q_{j}\right)\right) \\
& -\sum_{\sigma \in\left\{\sigma \in \Sigma \mid \rho_{\sigma, i} \geq \rho_{\sigma, j}^{\prime}\right\}} e \rho_{\sigma, i}^{\prime}\left(1-e d_{f p}^{n}\left(q_{i}, q_{j}\right)\right)
\end{aligned}
$$

(by the definition of $\phi_{q_{q}, q_{r}}^{n+1}$ and the induction hypothesis)

$$
\begin{aligned}
& =\sum_{\sigma \in\left\{\sigma \in \Sigma \mid \rho_{\sigma, i} \geq \rho_{\sigma, j}^{\prime}\right\}}\left(e\left(\rho_{\sigma, i}-\rho_{\sigma, j}^{\prime}\right)+e^{2} \rho_{\sigma, j}^{\prime} d_{f p}^{n}\left(q_{i}, q_{j}\right)\right) \\
& +\sum_{\sigma \in\left\{\sigma \in \Sigma \mid \rho_{\sigma, i}<\rho_{\sigma, j}^{\prime}\right\}} e^{2} \rho_{\sigma, i} d_{f p}^{n}\left(q_{i}, q_{j}\right) \\
& =e \sum_{\sigma \in\left\{\sigma \in \Sigma \mid \rho_{\sigma, i} \geq \rho_{\sigma, j}^{\prime}\right\}}\left(\rho_{\sigma, i}-\rho_{\sigma, j}+e \rho_{\sigma, j}^{\prime} d_{f p}^{n}\left(q_{i}, q_{j}\right)\right) \\
& +e \sum_{\sigma \in\left\{\sigma \in \Sigma \mid \rho_{\sigma, i}<\rho_{\sigma, j}^{\prime}\right\}} e \rho_{\sigma, i} d_{f p}^{n}\left(q_{i}, q_{j}\right) \\
& \left.=e d_{f p}^{n+1}\left(q_{q}, q_{r}\right) \text { (follows from }(3)\right)
\end{aligned}
$$

Next, the induction on the depth of formula is used to prove that $d_{L}\left(q_{q}, q_{r}\right) \leq e d_{f p}\left(q_{q}, q_{r}\right)$ by proving that $d_{L}^{n}\left(q_{q}, q_{r}\right) \leq e d_{f p}^{n}\left(q_{q}, q_{r}\right)$ for any $n \in \mathbb{N}$, where 


$$
d_{L}^{n}\left(q_{q}, q_{r}\right)=\sup _{\phi \in \mathcal{L}}\left\{\mid \phi\left(q_{q}\right)-\phi\left(q_{r}\right) \| \operatorname{depth}(\phi) \leq n\right\} .
$$

The base case is trivially satisfied. For $n \in \mathbb{N}$, assume:

$$
d_{L}^{n}\left(q_{q}, q_{r}\right) \leq e d_{f p}^{n}\left(q_{q}, q_{r}\right) .
$$

Then, according to Lemma 1 :

$$
\begin{aligned}
& d_{L}^{n+1}\left(q_{q}, q_{r}\right) \sup _{\phi_{\sigma}^{n} \in \mathcal{L}}\left\{\left|\bigvee_{\sigma \in \Sigma}\langle\sigma\rangle \phi_{\sigma}^{n}\left(q_{q}\right)-\bigvee_{\sigma \in \Sigma}\langle\sigma\rangle \phi_{\sigma}^{n}\left(q_{r}\right)\right|\right\} \\
&=e \cdot \sup _{\phi_{\sigma}^{n} \in \mathcal{L}}\left\{\sum_{\sigma \in \Sigma} \rho_{\sigma, i} \phi_{\sigma}^{n}\left(q_{i}\right)-\sum_{\sigma \in \Sigma} \rho_{\sigma, j}^{\prime} \phi_{\sigma}^{n}\left(q_{j}\right),\right. \\
&\left.\qquad \sum_{\sigma \in \Sigma} \rho_{\sigma, j}^{\prime} \phi_{\sigma}^{n}\left(q_{j}\right)-\sum_{\sigma \in \Sigma} \rho_{\sigma, i} \phi_{\sigma}^{n}\left(q_{i}\right)\right\} \\
&=e \cdot \sup _{\phi_{\sigma}^{n} \in \mathcal{L}}\left\{\sum_{\substack{\sigma \in \Sigma \\
0 \leq i \leq N-1}} \rho_{\sigma, i} \phi_{\sigma}^{n}\left(q_{i}\right)-\sum_{\substack{\sigma \in \Sigma \\
0 \leq j \leq N-1}} \rho_{\sigma, j}^{\prime} \phi_{\sigma}^{n}\left(q_{j}\right),\right. \\
&\left.\sum_{\substack{\sigma \in \Sigma \\
0 \leq j \leq N-1}} \rho_{\sigma, j}^{\prime} \phi_{\sigma}^{n}\left(q_{j}\right)-\sum_{\substack{\sigma \in \Sigma \\
0 \leq i \leq N-1}} \rho_{\sigma, i} \phi_{\sigma}^{n}\left(q_{i}\right)\right\}
\end{aligned}
$$

(as $G$ is deterministic)

where, for any $\sigma, \alpha \in \Sigma,\left|\phi_{\sigma}^{n}\left(q_{i}\right)-\phi_{\alpha}^{n}\left(q_{j}\right)\right| \leq d_{L}^{n}\left(q_{i}, q_{j}\right) \leq$ $e d_{f p}^{n}\left(q_{i}, q_{j}\right)$ (by induction hypothesis). In Pantelic and Lawford (2009), the function in (2) is a pseudometric (therefore, symmetry holds), and for $a_{\sigma, i}=\phi_{\sigma}^{n}\left(q_{i}\right)$, the constraints are satisfied, so $d_{L}^{n+1}\left(q_{q}, q_{r}\right) \leq e d_{f p}^{n+1}\left(q_{q}, q_{r}\right)$.

\section{3) Proof of Theorem 2}

Let $t$ be the string for which the supremum in (4) is reached. The formula corresponding to this distance is easily constructed. Assume that $t=\sigma_{1} \sigma_{2} \ldots \sigma_{n}$. Then, the formula is given as $\phi=\left\langle\sigma_{1}\right\rangle\left\langle\sigma_{2}\right\rangle \ldots\left\langle\sigma_{n}\right\rangle$.

4) Proof of Theorem 3

Similar to Theorem 2, by using the disjunction formula.

5) Proof of Theorem 4

Since $L_{p}\left(G_{1}\right)=L_{p}\left(G_{2}\right), G_{1}$ and $G_{2}$ are probabilistic traceequivalent in the sense of Jou and Smolka (1990). As $G_{1}$ and $G_{2}$ are deterministic, probabilistic trace-equivalence implies probabilistic bisimulation equivalence. Therefore, $d_{f p}\left(G_{1}, G_{2}\right)=0$.

6) Proof of Theorem 5

Follows from the construction of $G_{1 a}$.

7) Proof of Theorem 6

Let $G_{2}=\left(Q_{2}, \Sigma, \delta_{2}, r_{0}, p_{2}\right)$, where $p_{2}$ is given by (6). Also, let $G_{2}^{*}=\left(Q_{2}, \Sigma, \delta_{2}, r_{0}, p_{2}^{\prime}\right)$ be a probabilistic generator with an arbitrary probability distribution $p_{2}^{\prime}$. We use induction to show that $d_{f p}\left(G_{1 a}, G_{2}^{*}\right) \geq d_{f p}\left(G_{1 a}, G_{2}\right)$ by showing that $d_{f p}^{n}\left(G_{1 a}, G_{2}^{*}\right) \geq d_{f p}^{n}\left(G_{1 a}, G_{2}\right), n \in \mathbb{N}$. For $q \in Q_{2}$, let $d_{f p}^{n}(f(q), q)$ be the distance between the states $f(q)$ of $G_{1 a}$ and $q$ of $G_{2}$, and $d_{f p}^{* n}(f(q), q)$ be the distance between $f(q)$ of $G_{1 a}$ and $q$ of $G_{2}^{*}$. The base case is trivially satisfied. Next, assume that, for each $q \in Q_{2}$, $d_{f p}^{* n}(f(q), q) \geq d_{f p}^{n}(f(q), q)$. The functions $i$ and $j$ are defined as for (5), and, for $q \in Q_{2}, k(q, \sigma)=k$ such that $r_{k}=\delta_{2}(q, \sigma)$ if $\delta_{2}(q, \sigma) !$, and $k(r, \sigma)=0$, otherwise. The short notation $k$ will be used. For $q \in Q_{2}$, let $\rho_{f(q)}, \nu_{q}$ and $\nu_{q}^{\prime}$ be the distributions induced by the states $f(q)$ of $G_{1 a}$, $q$ of $G_{2}$ and $q$ of $G_{2}^{*}$, respectively. Also, for $q \in Q_{2}$, let $\rho_{\sigma, i}$ be used instead of $\rho_{f(q)}\left(\sigma, q_{i}\right)$, and, similarly, $\nu_{\sigma, j}$ instead of $\nu_{q}\left(\sigma, r_{j}\right)$ and $\nu_{\sigma, k}^{\prime}$ instead of $\nu_{q}^{\prime}\left(\sigma, r_{k}\right)$. Then:

$$
\begin{aligned}
& d_{f p}^{* n+1}(f(q), q) \\
& =\sum_{\sigma \in\left\{\sigma \in \Sigma \mid \rho_{\sigma, i} \geq \nu_{\sigma, k}^{\prime}\right\}}\left(\rho_{\sigma, i}-\nu_{\sigma, k}^{\prime}+e \nu_{\sigma, k}^{\prime} d_{f p}^{* n}\left(q_{i}, r_{k}\right)\right) \\
& +\sum_{\sigma \in\left\{\sigma \in \Sigma \mid \rho_{\left.\sigma, q_{i}<\nu_{\sigma, k}^{\prime}\right\}} e \rho_{\sigma, i} d_{f p}^{* n}\left(q_{i}, r_{k}\right)\right.} \rho_{\sigma \in \operatorname{Pos}(f(q)) \backslash \operatorname{Pos}(q)} \rho_{\sigma, i} \\
& +\sum_{\sigma \in\left\{\sigma \in \operatorname{Pos}(q) \mid \rho_{\sigma, i} \geq \nu_{\sigma, k}^{\prime}\right\}}\left(\rho_{\sigma, i}-\nu_{\sigma, k}^{\prime}+e \nu_{\sigma, k}^{\prime} d_{f p}^{* n}\left(q_{i}, r_{k}\right)\right) \\
& +\sum_{\sigma \in\left\{\sigma \in \operatorname{Pos}(q) \mid \rho_{\sigma, i}<\nu_{\sigma, k}^{\prime}\right\}} e \rho_{\sigma, i} d_{f p}^{* n}\left(q_{i}, r_{k}\right) \\
& \geq \sum_{\sigma \in \operatorname{Pos}(f(q)) \backslash \operatorname{Pos}(q)} \rho_{\sigma, i}+\sum_{\sigma \in \operatorname{Pos}(q)} e \rho_{\sigma, i} d_{f p}^{* n}\left(q_{i}, r_{k}\right) \\
& \sum_{\sigma \in \operatorname{Pos}(f(q)) \backslash \operatorname{Pos}(q)} \rho_{\sigma, i}+\sum_{\sigma \in \operatorname{Pos}(q)} e \rho_{\sigma, i} d_{f p}^{n}\left(q_{i}, r_{k}\right)
\end{aligned}
$$

(because of induction hypothesis, since $q_{i}=f\left(r_{k}\right)$ )

$$
\begin{aligned}
& =\sum_{\sigma \in \operatorname{Pos}(f(q)) \backslash \operatorname{Pos}(q)} \rho_{\sigma, i} \\
& +\sum_{\sigma \in \operatorname{Pos}(q)} \max \left(\rho_{\sigma, i}-\nu_{\sigma, j}+e \nu_{\sigma, j} d_{f p}^{n}\left(q_{i}, r_{j}\right), e \rho_{\sigma, i} d_{f p}^{n}\left(q_{i}, r_{j}\right)\right) \\
& \left(\operatorname{since} \nu_{\sigma, j} \geq \rho_{\sigma, i} \text { for every } \sigma \in \operatorname{Pos}(q)\right) \\
& =d_{f p}^{n+1}(f(q), q)
\end{aligned}
$$

